\title{
The Immune System and Othep Cognitive Systems
}

In the following pages we propose a theory on cognitive systems and the common strategies of perception, which are at the basis of their function. We demonstrate that these strategies are easily seen to be in place in known cognitive systems such as vision and language. Furthermore we show that taking these strategies into consideration implies a new outlook on immune function calling for a new appraisal of the immune system as a cognitive system.
URI HERSHBERG AND SOL EFRONI

\section{Uri Hershberg is at the}

Interdisciplinary Center for Neuronal

Computation, at the Hebrew

University, Jerusalem, Israel. Sol Efroni is at the Computer Science and Applied Math Department and at the Immunology Department at the Weizmann Institute of Science, Rehovot, Israel.

E-mail for communication: uriher@cc.huji.ac.il. $t$ is becoming clear that the field of immunology is approaching a paradigm shift. It is agreed by most researchers that the immune system is a complex system both in its composition and its behavior. However, the most popular ideas of immune function treat the immune system in a mechanistic and reductionist manner. According to the clonal selection theory the immune system's function is to defeat pathogens. The immune system identifies foreign antigens and destroys them. The identification of the foreign is made possible by removing, in the immune system's prenatal development, all receptors that recognize self. Anything that an immune receptor identifies "must be the enemy" [1]. Countering this mainstream view are a growing number of voices that state the need to change the clonal selection theory or discard it, claiming that such a simplistic appraisal of the immune system's function and mode of action is untenable because, at the molecular level, we are closely related to the pathogens that invade us. There is a need to consider the immune system as a integrative system with the ability to see patterns and understand context [2,3]. It is in the context of this argument about the immune system that we present our theory of cognitive systems and claim that the immune system should be seen as such a cognitive system.

The phrase "cognitive system" is used in many fields to describe the various faculties that we and other organisms use to perceive and interact with the world. Despite its widespread use, the phrase "cognitive systems" has not yet been defined in a way that can be applied to all of the cases in which it is used.

We suggest the following criterion to differ between cognitive and noncognitive systems: In cognitive systems the perceptual sensitivities of the system are not preordained only by the plan of the system but need an interaction with their environment to define the system's exact sensitivities. 
We propose a theory of the general underlying principles of cognitive systems, their perception regarding the environment, and the way in which they deal with the complex patterns their environments present. This is essentially divided into two phases-a phase of priming (top of Figure 1), in which the system defines the general properties of its environment that it knows and a phase of specific interaction (bottom of Figure 1), in which the cognitive system utilizes its knowledge of the general properties to interact with specific encounters with its environment. These phases are not necessarily chronological by nature and happen with each interaction of the cognitive system with its environment.

ognitive systems are innately built with a tendency toward perceiving certain aspects of the environment. These tendencies are such that they cause the cognitive systems to be receptive toward seeing certain general properties of the environment and examples that embody them. However, the cognitive system will only acquire general properties that are corroborated in its initial interactions with the environment. The definition of general properties by cognitive systems is something that is not completely predetermined but rather defined through interaction with the environment; the environment's reinforcement is what defines the final set of general properties that the cognitive system uses to know its environment. The general properties are an important aspect of the shape space, ${ }^{1}$ and so they are encountered often in meaningful interactions with the shape space. Examples of such properties which are both generic for and

${ }^{1}$ Protein shape space is an analogy commonly used to describe a vector space, in which every point describes a configuration of the protein [4]. We will use this in a more general way to describe the vector space of the various cognitive modalities.

\section{FIGURE 1}

The Two Phases Enabling Cognitive Perception of Complex Patterns:

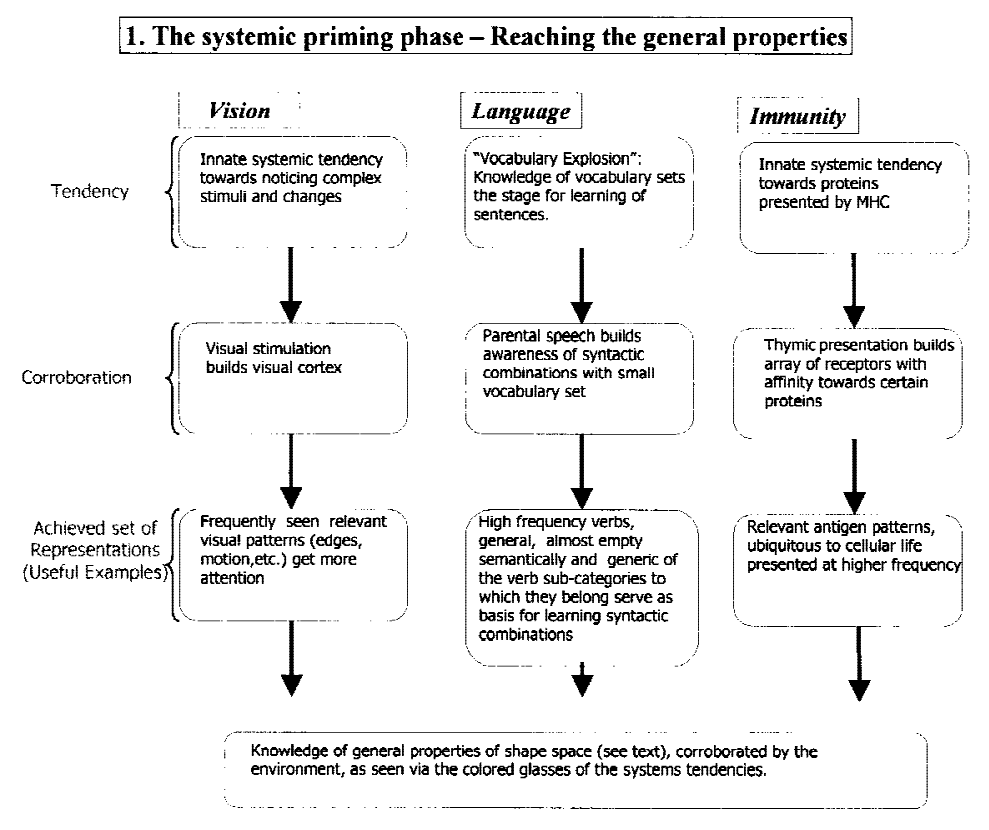

2. Specific interactions with the environment

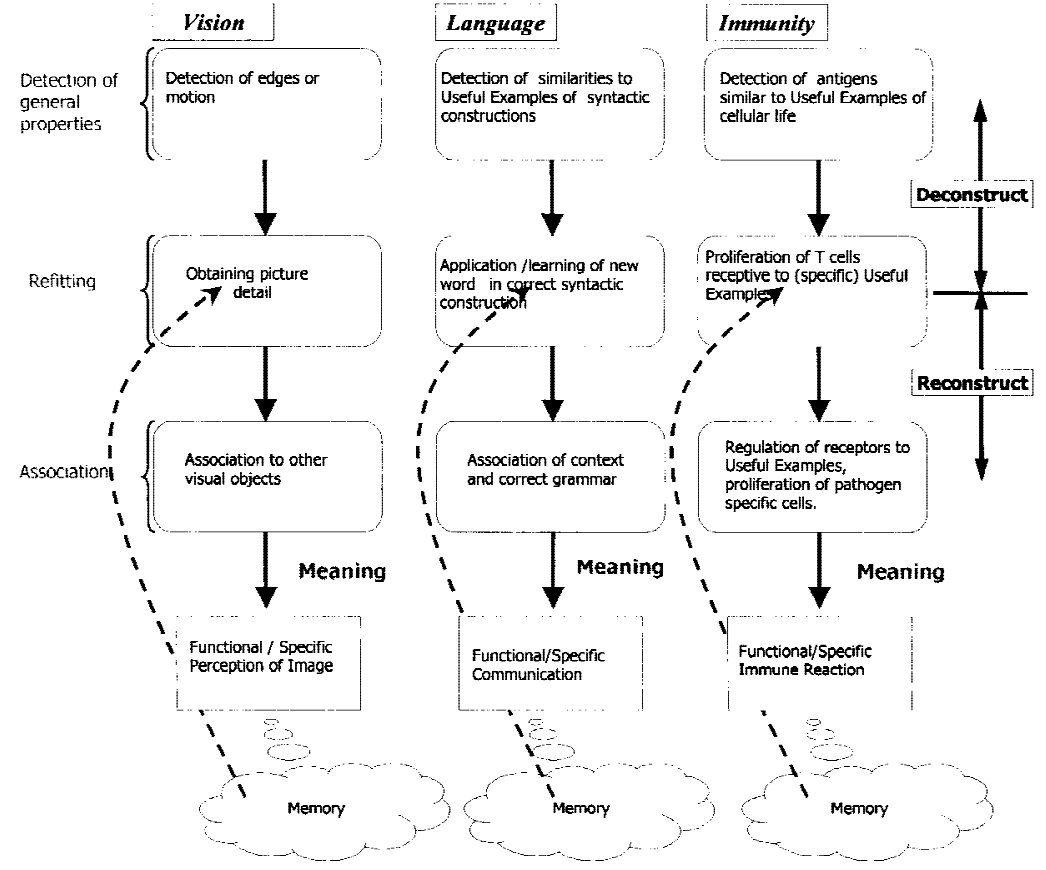

In the above figure we explain our theory on cognitive systems, utilizing for this purpose examples from vision, language and the immune system. The figure shows the two phases encompassing cognitive perception. 
ubiquitous to the relevant environment, are extremely beneficial in learning the environment. As such we will call them "useful examples."

Through specific interactions and based on the previous tendencies of the system certain general properties are corroborated by "useful examples" of these properties appearing in the environment. This corroboration leads to the formation of an achieved set of representations in the system. Specific encounters with the environment start with a deconstruction via a detection of similarities to the useful examples, embodied in the achieved set. After this detection there is a refitting and fine tuning to the specific elements of the event. Having identified the specific elements of the event, there is now a reconstruction of the event together with an association to other contextual factors to a functional/meaningful event that can be appropriately reacted to and added to the memory of the system. [The two phase are chronologically mingled; both happen simultaneously. Every encounter is a specific encounter even while the cognitive system is still building its understanding of the general properties. Also in many cognitive systems it is not clear if the process of defining new general properties ever comes to a complete stop. "Young" cognitive systems are less fluent in working with the general properties, and it is harder to teach an "old" cognitive system new tricks, but all interactions with the environment have elements of both phases.]

$\mathrm{n}$ this discussion of cognitive systems, we will start with the visual system. Visual systems are built according to the niche in which they are used. A bee's view of flowers is different from ours. The limits of sensitivity to wavelength and contrast are to a great extent built in. However, seeing is not merely a matter of light sensitivity. We see because we have learned how to do so; we know what things to "look" for and what they mean. This knowledge is an understanding of the natural context of vision and the general properties of the visual stimulus that we encounter.
As an example, take a look at Figure 2 where our knowledge will not help us.

A painting from the brush of Salvador Dali, this picture shows how complicated it is to know what we are looking at when we do not have the natural context to tell us what we should look for. The title may give you a hint, "Apparition of a Face and Fruit Dish on a Beach." Once we reveal that the "apparition" hides the figure of a dog, it is doubtful if you could ever again look at this picture without seeing man's best friend. Our preknowledge is not of specific aspects of every stimulus but rather of their general properties. The knowledge of the general properties of visual stimuli is not something that we are born with; rather, it is acquired through the natural exposure to the environment. The process starts with the natural visual tendencies of the visual apparatus and the innate bias that we are born with toward certain things, such as complex stimuli and movement; however, these innate aspects are not enough. Given this tendency, the visual system, in its first stages of development, builds up the tools that later will allow the act of vision.

The need for this priming stage and the existence of a critical period for its occurrence is easily seen in people who have had blocked corneas from infancy.
Such people, if their cornea is removed too late, will remain functionally blind; for although they can react to light, they cannot resolve the images they see [5] (much like living in a painting by Dali). In those of us free to see the world, the process of learning the general properties is unsupervised and is based on the existence of useful examples for the general properties of naturally encountered visual stimuli.

The general properties are those things that are both ubiquitous to the different stimuli and appear in many different meaningful contexts. In vision these would be things such as edges or regularities of scale [6]. Their high frequency and usefulness very naturally cause their corroboration, until eventually we have a visual system with an achieved set of representations based on the "useful examples" (stage 1 in Figure 1). This "achieved set" is what enables, from then on, the resolution of visual stimuli: the deconstruction and reconstruction of the second stage, which allows the acquiring of the proper functional understanding of the image and memory.

Vision starts with the general properties of the image rather than its particulars. In our representation this is the phase of detection (second half of Figure 1). Sight, as opposed to taking a picture,

\section{FIGURE 2}

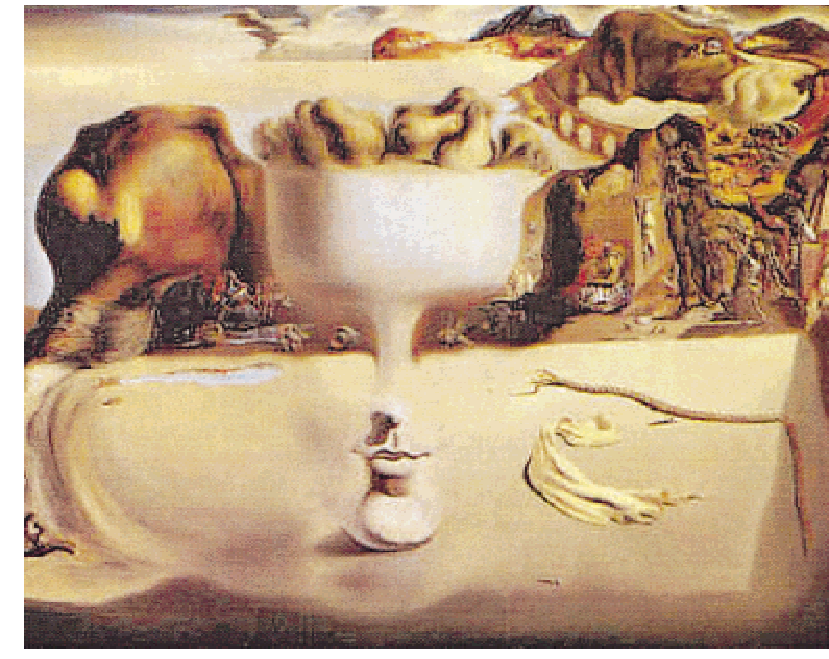

“Apparition of a Face and Fruit Dish on a Beach": Salvador Dali (1970). 
is done while knowing what the important things in the image are. Edges, movement, and other "eye catching" things tell the visual system where to look. Once this phase of detection and deconstruction is done, the visual system can refit to the particular image it is dealing with and can associate it to the current context. This gives the stimulus meaning and achieves a functional image, while widening the knowledge of the visual world via memory.

The fact that our visual ability is based on and inseparable from our perception of the general properties of visual stimuli can easily be seen in the kinds of illusions that fool us. Movies, animation, and paintings are all only good copies of natural images in the sense that they capture the general properties of the visual world.

Note the different scales involved in the two phases that we have discussed so far regarding vision. The first stage, the stage of priming and obtaining the achieved set, is a stage that responds to some external input, but does so in a duration orders of magnitude longer than the time needed to process new input once the system is fully functional. Moreover, the second stage, specific interaction, is a continuous process going on all the time, whereas the first stage is a once in a lifetime opportunity. It takes a long time to put together an organism, but, once it exists, the organism has to use its mechanisms immediately.

$\mathbf{0}$ ne cognitive system where this separation in time is clearly seen is in the development of language in children. As infants and children, we learn to speak, and it is a long and laborious process. However, once language is acquired, when we add new words to our vocabulary, learning them and their correct use is something that we can do almost instantaneously. We will deal with only one aspect of the acquisition of language - the learning of correct syntactic combinations-how we know the correct order of words that make a meaningful sentence. This step is made possible by the previous stages of auditory and cognitive development, which are influenced by various innate and learned biases. The exact amount of learned and innate influence is the scene of many an argument in the cognitive sciences that we will not go into [7]. In any case, it is obvious that at the stage of syntactic development the language system already has a "tendency" that sets the stage for the learning of syntactic combination. This can be seen in the fact that the learning of sentence formation is always preceded, in children, by a stage of rapid growth in vocabulary or "vocabulary explosion," that brings about the creation of the vocabulary necessary for syntactic combinations [8].

In studies concerning the use of intransitive and transitive verbs in syntactic combinations, in parents' conversations with their children, it was seen that parents use a very small subset of verbs at a very high frequency when talking to their children. Words such as want, come, go, and make account for a high fraction of the verbs used in parental conversation. All these highfrequency verbs are very general, have uses that are almost empty semantically, and can be said to be generic of the verb subcategories to which they belong. In return, all the first verbs used by children are drawn from this group of verbs (though individually each child's first verb need not be the most commonly said word of his parent). Further, once the first verbs are learned in a certain syntactic construction, the speed of learning other verbs in the same syntactic construction, but not necessarily in other constructions, is greatly enhanced. This could be indicative of a scenario where the child learns the first 2 or 3 examples, after which the others are greatly facilitated (Reference 9 and Figure 3).

In effect, because of the shape space of languages, in the course of normal conversation, children are exposed to the useful example of the different types of syntactic combinations and the correct use of language. The first words are very common and have many uses (they embody various ideas). In this fashion the useful examples of language are corroborated, bringing about the formation of an achieved set of representations that greatly facilitates the latter identification of similarities to the use-

\section{FIGURE 3}

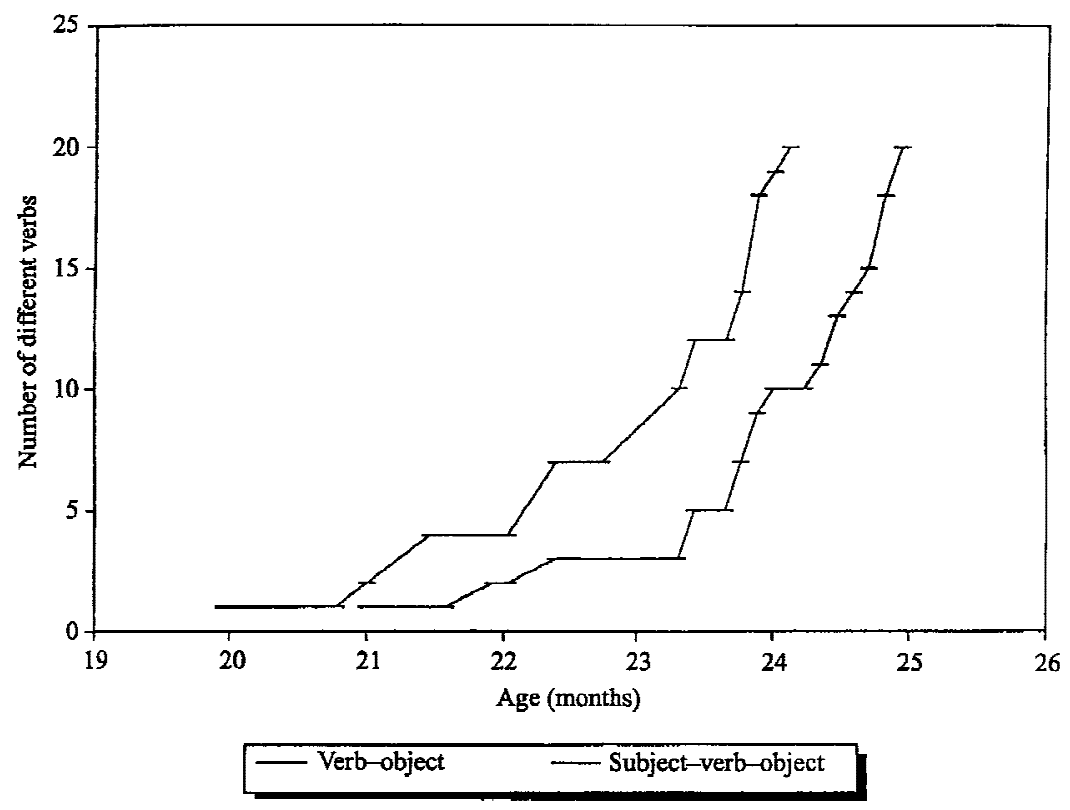

Figure 3. Cumulative number of different verbs in V0 and SVO word combinations produced by a subject as a function of age [9]. 
ful examples and the formation of correct functional syntactic combinations.

The studies mentioned above deal with the learning of intransitive verbdependent combinations and of transitive verbs in verb-object (VO) and subject-verb-object (SVO) relations. The view of the first examples as useful examples is further strengthened by the fact that the first words learned are easily seen to be generic of some relation of the speaker to the world. In learning intransitive verbs children deal with animate objects, and the first few verbs spoken in word combinations (i.e., learned) always cover categories both of active (come, go) and passive/static (fall, sleep) relations [9]. VO syntactic combinations are learned before SVO. The improvement in one syntactic combination is separate from the improvement in the other. In 13 of 16 children, the Hebrew verb "raza" (want) was one of the first VO verbs (and if not, it was generally the first SVO verb). In VO, $84.4 \%$ of first utterances were requests, whereas in SVO $25.9 \%$ were requests and $44.4 \%$ were descriptions of creation or consumption of objects. In learning both syntactic combinations what is being learned, along with the correct use of VO and SVO, are useful examples of the child's relation to alienable objects (things that can be part of me or not part of me). The child is learning to add, remove, or maintain objects in his "personal space" [10] and in essence is acquiring an achieved set of representations to deal with objects and their abstract reflections.

The statistical shape of language is such that when speaking to those who do not speak, we will use those words that are useful examples and enable the cognitive task of learning. Languages are "built" so that when talking in simple language to children, we will corroborate the general properties of correct speech and sentence formation, thus enabling the acquisition of language. Possibly, we have here also evidence for the priming phase of the most cognitive of modalities: our ability to exercise abstract thoughts. Together with the general properties of language, we see here that children learn useful examples of the mental interaction with alienable objects and define some of the general properties of abstract thought.

H aving shown how our theory works in systems, commonly agreed upon to be cognitive, we come now, finally, to the immune system. It is in treating the immune system as cognitive that we believe that our theory is most controversial and also of the greatest benefit compared with the present paradigm, the clonal selection theory. We hope to show, citing various sources of contemporary research, that present knowledge of the immune system and its interaction with the antigenic/molecular patterns of our body calls for the treatment of the immune system as a cognitive system.

The immune system has an ability to identify specific events and changes in the body. The immune system's environment is the body. It interacts on the cellular/molecular level. To do this, it has many types of cells as well as effector and signaling substances, many of which are yet to be identified and understood. However, in general the population of cells that make the immune system can be characterized as the populations of cells known as lymphocytes. The two most important groups of lymphocytes are called B cells and $\mathrm{T}$ cells.

Both of these cell families have a unique ability the create receptors, which, though they all originate from the same genetic material, use different combinations of this material to create an immense variability in their final form. The shape of the receptor, which like all proteins is based on the sequence of a certain gene, implies the shape and type of molecule that will activate the receptor. Therefore, this genetic variability gives the immune system the potential ability to have receptors that can identify a near infinite number of molecular shapes. The molecules that immune receptors identify are commonly known as antigens. The region within the antigen to which they attach is known as an epitope. A single antigen may have several different epitopes.
The receptors of B cells identify extracellular substances. The receptors of $\mathrm{T}$ cells identify intracellular substances by interacting with specialized antigenpresenting proteins known as major histocompatability complex (MHC) receptors [11], which are expressed on the surface of every one of the body's cells. MHCs present fragments of intracellular proteins, in effect mirroring the internal state of the cell. Together, T cells and B cells can identify most intra- and extracellular substances. The immune system's identification and reaction to a pathogen or other immune events is dependent on mutual reaction by both $\mathrm{T}$ cells and B cells to that event [12].

In trying to fit the immune system to our theory of cognitive systems, we are making a remark on the kind of receptor repertoire that the immune system forms out of its potential variability.

The potential repertoire of receptors is immense, between $10^{11}$ for $\mathrm{B}$ cells and $10^{16}$ for $\mathrm{T}$ cells [4]. Because (in mice) the immune system contains only about $10^{8}$ of each of the types of cells and every single cell has only one type of receptor, it is obvious that the actual repertoire is smaller. If the immune system were to have a repertoire built of every potential receptor it can generate, then in a rat, for example, this would necessitate having a spleen 70 times the size of the rat's entire body [13]. What kind of repertoire actually exists, and what factors are important in its formation?

As we mentioned above, immunology is in the midst of a paradigm shift. There is an especially widespread debate on the way in which the immune system differentiates between the molecular patterns of the body and foreign pathogens [3]. We will not go into all aspects of this discussion. In plain words, the current textbook outlook on immunity, the clonal selection theory, states that anything that an immune cell receptor identifies is a foreign pathogen. According to the clonal selection theory, this state of affairs is brought about in the following way: During embryonic development immune cells are created randomly, each reactive to a different antigen. Those 
cells bearing receptors that bind to selfantigens at a certain level of affinity or above are eliminated. This is known as negative selection. At the end of this process any receptors that remain can only be activated by foreign pathogens $[1,14]$.

This is a classical reductionist theory that is extremely elegant and simple. It explains how we create a repertoire, which on the one hand can identify pathogens and yet does not react falsely with our body's molecular patterns. It is also a very mechanistic way of viewing the workings of the immune system. One major implication of this theory is that autoimmunity - the reaction of immune receptors to self antigens-is something that exists only as a pathology and never in a properly functioning immune system or a healthy body. Even if we were not about to show that the immune system works like a cognitive system, there is a major problem with this theory. The elimination of selfrepertoire completely ignores other functions of the immune system that are essentially involved with self, such as wound healing and combating cancer [2,15].

Several generally known aspects of immune detection, agreed on even by the most ardent supporters of the clonal selection theory, seem to imply that the immune system is working as a cognitive system: First, the need for costimulation of $\mathrm{B}$ cells and $\mathrm{T}$ cells for immune reaction [12], and second, the fact that $\mathrm{B}$ cells are reacting to extracellular information, whereas $\mathrm{T}$ cells react to intracellular information. Together these appear to imply an immune reaction to patterns and context.

Treating the immune system as a cognitive system, the idea of building a repertoire in the way suggested by the clonal selection theory becomes less plausible. The immune system's environment is built completely of cells both endogenous and exogenous, which at the time of encounter are residing in the body. Also, all of this cellular and viral life is built of similar building blocks. There is no intrinsic molecular signal that differentiates between the organic substances of our body and those of other organisms. Removing all receptors to self amounts to removing all receptors to all of the things that are common to all cellular life. Building a system that needs to recognize the important aspects of this environment but is blind to the general properties (which are those things that are ubiquitous in the environment) is like building a human visual system that can not become aware of edges.

We are suggesting a model of the immune system as a cognitive system. This implies several things about the way the immune system is primed and how it detects its environment (see Figure 1). As we showed for vision and language, the priming of the system and building of the achieved set of representations starts by fulfilling innate systemic biases or tendency. In this case, this would probably be a genetically transferred tendency to present certain protein examples that are used to build the receptor repertoires. These useful examples would, as in vision and language, be examples of the general properties of the living molecular environment. They should, therefore, be examples of self that cause a positive selection of receptors with at least some minimal affinity to these examples.

We would, therefore, expect to find that, from the first randomly generated stock of receptors, the adult repertoire of immune receptors are created by a combination of positive and negative selection using specific molecular examples of self.

Part of the reason that the clonal selection theory is being forced to change is that positive selection is apparently important for the creation of the mature repertoires in both $\mathrm{B}$ cells and $\mathrm{T}$ cells $[16,17]$. This is especially evident in $\mathrm{T}$ cells. One form of positive selection is agreed on for $\mathrm{T}$ cells, even by the clonal selection theory. $\mathrm{T}$ cells must have a minimal affinity for at least one self antigen-MHC receptors-if they are to function. However it appears that the recognition of self-MHCs is not the only kind of self-recognition that is necessary for proper T-cell development, in fact the proteins nested in the MHCs, while selection is in process, affect the positive selections outcome [18]. Further, it has been shown, using genetic engineering techniques, that an immune system built with fewer kinds of fragments presented by MHCs, within the context of positive selection, will have a less diverse T-cell repertoire [18]. The selection of $\mathrm{T}$ cells within certain boundaries of affinity to MHC receptors and the fact that MHC receptors only present the fragments of certain proteins [11], together show a possible mechanism by which the immune system creates an important bias toward a certain population of examples while creating the repertoire of receptors.

What are the "useful examples" presented by the MHCs bias? The exact types of proteins, fragments of which are presented by MHC, have not yet been characterized. However, let us consider, given a tendency, what would constitute the useful examples that are corroborated, and what is the environment for which they create an achieved set? As we mentioned before, the environment of the immune system is the body's cellular life. Candidates for "useful examples" would have to have the following properties: they would have to be part of every cell; they would have to have been there all through the history of the development of the immune system for them to be such a major part of its function; they should be relevant in times of stress (or otherwise they cannot serve extreme conditions usually common in immune response).

Indeed, it is possible to find such a set. The set corresponds to a group of antigens Cohen has named "homuncular antigens" [2], and all belong to a group called housekeeping or maintenance proteins. Housekeeping proteins are essential in all cells, because they are responsible for ongoing energy metabolism, protein construction, and basic genetic manipulations.

One group of housekeeping proteins of special interest is called heat shock proteins (HSPs). HSPs are part of a larger group of proteins called chaperones, which are essential in correct protein construction and folding. HSPs help cells maintain the proper form (and function) of proteins in various 
states of emergency and stress. The situations when HSPs are most expressed, emergency and stress, are also the ones where you may usually find immune response. That alone suffices to mark HSPs as useful examples to the immune system, but more than that, because states of emergency abound in all environments, HSPs are essential and are expressed in all cells. In fact, these proteins are ubiquitous in cells in times of stress and are highly preserved throughout evolution, from prokaryotes to multicellular organisms [19]. All this suggests HSPs to be part of the useful examples that build the achieved set through which detection is carried out.

Now that we have proposed this possible achieved set (the homuncular antigens), we can go on to the mechanism of detection. If the immune system behaves according to our suggested theory, we would expect several points of immune behavior. We have just shown that housekeeping genes in general and HSPs in particular would be good candidates as "useful examples," which would imply that the immune system and its receptors would have a bias in its reaction to these proteins when reacting to the environment and performing immune functions. At least in HSPs this appears to be the case. Receptors to different types of endogenous and exogenous HSPs have been shown to be important to immune reaction. In Cohen and Young's review of the immunological reaction to HSPs, they show that during almost any reaction to bacteria and parasites, the recognized antigen detected is a type of HSP [20]. In other immune activities, T cells reactive to specific self-proteins, for example, HSP60 and MBP (an essential factor in nervous tissue), have been shown to enhance the regeneration of skin and nerve tissue [21-24].

If the immune system is indeed working as a cognitive system, as we have described it, then the immune mechanisms of detection should include a means for the detection of the general properties of the environment along with a mechanism for refitting toward specific encounters. This should allow for the deconstruction and recon- struction of the immune "image." Therefore, we are expecting an adult repertoire of receptors built to react degenerately, at a median level of affinity, to a few self-antigens. This repertoire will not change much during our lifetime. Along side this, we expect a repertoire of non-self-reactive receptors, which changes and evolves over time as the immune system encounters pathogens and evolves throughout our life.

Both $\mathrm{T}$ cells and $\mathrm{B}$ cells have adult receptor repertoires that are highly cross-reactive and react degenerately to self-antigens. These repertoires remain at a steady level throughout life [13,25]. B cells have an added ability: when activated by the innate immune repertoire, they start a process of fine tuning, known as affinity maturation [26], by which they create highly specific and accurate receptors that react to a specific antigens. In B cells we find a sharp distinction between a self-reactive repertoire, which remains permanent throughout life, and a changing population of receptors that is a result of the immune system's interactions with different immune events [25]. B cells, while acquiring a growing repertoire toward specific pathogens, during the lifetime of the organism, maintain a permanent repertoire of cross-reactive receptors, possibly through interaction with the less changeable T-cell repertoire.

The stability of T-cell repertoires and fluidity of B-cell repertoires could very well be a way to allow the process of detection and refitting, while keeping focused on the achieved set of molecular life (homuncular antigens).

The existence of self-reactive receptors does not mean that an autoimmune reaction of the immune system is something that happens in health. It means that such an occurrence is avoided, not through the lack of self-reactive immune receptors, but rather through their heavily controlled existence.

Although we have not described exact cellular mechanisms of detection and interaction, we feel that we have given enough of an argument to justify treating the immune system as a cognitive system.

The immune system's ability to function is dependent on its understanding the environment in a cognitive manner and its ability to discern the complex patterns it encounters. The world of immune function is the body in which it resides. This is the source of the examples it uses to see the generalities of cellular life. These points imply a new outlook at the receptor repertoire of the immune system, which enables it to react to changing and unexpected patterns.

Our self is the source of examples, and yet in the end the immune system knows not to react to the patterns of self. This could be because the immune system has no receptors for self and so the immune system can not "see" it. We would suggest that this is not so, but rather the immune system has many receptors with a sensitivity for self; however, their interaction is such that it recognizes the patterns of self as what they are-the background on which the picture of immune events are painted. Selfimmunity is the basis not the nemesis of immune function.

( n closing, although we have used vision and language as examples to explain something about the immune system, still in doing so we have also put together principles that are at the base of all cognitive systems. These three systems are all different in their particular components and in the specific fashion in which they interact with their part of the environment. Still they all share the common traits of dealing with the specifics of their environment through an acquired sensitivity to the general properties of their environment. We would say that this is what makes them all cognitive systems.

Cognitive systems have the common trait of functioning in an existing environment constantly on the brink of change. Noncognitive systems are built with a limited hardwired perceptual ability capable of reacting to certain stimulants and no others. In cognitive systems, the existence of a built in (genetic) tendency promises the acquisi- 
tion of generalities proven to be important over several evolutionary steps. However, because of the need for corroboration, the cognitive system retains an ability to change, over time, the definition of the generalities with the changing of the environment. The ability to modify the innate tendencies and the need to build the understanding of general properties relative to the environment for every generation is what sets apart the function of cognitive and noncognitive systems.

Cognitive understanding deals with specific things and the ability to recognize a great amount of the specific aspects of its environment. However, the ability to deal with all of the varying and changing aspects of the environment starts not from the specifics of the world but from generalities. First are learned the general metaphors, the useful examples of general properties, which are applicable in many different ways to the world. Once they are grasped, it is easy to apply them in many different ways to the world around us. The fact that generalities take on many different aspects is not a drawback to learning them. It is one of the main reasons they are learned first. We have dealt here with only three systems and only in a superficial manner; still, we are sure that studying other cognitive systems will show similar patterns. Furthermore, we believe that in studying cognitive sys- tems discovering the nature of their useful examples and the general properties they represent will tell us much as to those systems basic function and abilities.

\section{ACKNOWLEDGMENTS}

We thank Professor Irun Cohen, of the Immunology Department at the Weizmann Institute in Rehovot, and Professor Anat Ninio, of the Psychology Department at the Hebrew University in Jerusalem. Without their insights and help, none of these ideas would have started forming, nor could they have reached their final shape. This research was supported by a grant from the Ministry of Science, Israel.

\section{REFERENCES}

1. Burrnet, F.M. A modification of Jerne's theory of antibody production using the concept of clonal selection. Aust J Sci 20, 1957, 67-69.

2. Cohen, I.R. Tending Adam's garden. Academic Press, San Diego, CA, 2000.

3. Seminars In Immunology. Vol. 12, No. 3, (June 2000, R.E. Langman, M. Cohn, eds.).

4. A.S. Perelson, and G. Weisbuch. Immunology for physicists. Rev. Mod. Phys. 69/4, 1997, pp. 1219-1267.

5. Clyde, W.0. The human eye. Sinauer, Sunderland, MA, 1999.

6. Cutting, J.E. Perception with an eye for motion. MIT, 1986.

7. Ingram, D. Child Language Acquisition: Method, description, and explanation. Cambridge University Press, 1989.

8. Bates, E., Bretherton, I., Snyder, L., and Beeghly, M. From first words to grammar: individual differences and dissociable mechanisms. Cambridge University Press, 1988.

9. Ninio, A. Model learning in syntactic development: Intransitive verbs. International Journal of Bilingualism, 3, 111-131. (Invited article for a special issue on Cross-linguistic Studies of Early Grammar, edited by M.M. Vihman, 1999).

10. Ninio, A. Pathbreaking verbs in syntactic development and the question of prototypical transitivity. Journal of Child Language. 26/3, 1999, pp. 619-653.

11. Germain, R.N., Stefanova, I. The dynamics of T cell receptor signaling: complex orchestration and the key roles of tempo and cooperation. Annu Rev Immunol 17: 1999, pp. 467-522.

12. Langman, R.E., and Cohn, M. A minimal model for the self/non-self discrimination: a return to the basics. Seminars in Immunology 12(3): 2000, pp. 189-195.

13. Mason, D. A very high level of crossreactivity is an essential feature of the T-cell receptor. Immunology Today 19(9): 1998, 395-404.

14. Janeway, C.A., Travers, P., Walport, M., and Capra, J.D. Basic concepts in immunology. Immuno-biology: the immune system in health and disease. Chapter 1, especially Figure 1.13. (4th ed., 1999).

15. Paul, W.E. Tumor immunology: effector mechanisms in cancer immunity. Fundamental Immunology Chapter 37 (4th ed., 1998).

16. Hayakawa, K., Asano, M., Shinton, S.A., Gui, M., Allman, D., Stewart, C.L., Silver, J., Hardy, R.R. Positive selection of natural autoreactive B cells. Science 285(5424): 1999, 113-116.

17. Sebzda et al. Selection of the T cell repertoire. Annu Rev Immunol 17, 1999, 829.

18. Barton, G.M., Rudensky, A.Y. Requirement for diverse, low-abundance peptides in positive selection of T cells. Science 283(5398): 1999, 67-70.

19. Gupta Redhay, S. Protein phylogenies and signature sequences: a reappraisal of evolutionary relationships among archaebacteria, eubacteria, and eukaryotes. Microbiol Mol Biol Rev 62(4): 1998, 1435-1491.

20. Cohen, I.R., Young, D.B. Autoimmunity, microbial immunity and the immunological homunculus. Immunol Today 12(4): 1991, 105-110.

21. Moalem, G., Yoles, E., Leibowitz-Amit, R., Muller-Gilor, S., Mor, F., Cohen, I.R., Schwartz, M. Autoimmune T cells retard the loss of function in injured rat optic nerves. J Neuroimmunol 106(1-2): 2000, 189-197.

22. Schwartz, M., Cohen, I.R. Autoimmunity can benefit self-maintenance. Immunol Today 21(6): 2000, 265-268.

23. Abulafia-Lapid, R., Elias, D., Raz, I., Keren-Zur, Y., Atlan, H., Cohen, I.R. T cell proliferative responses of type 1 diabetes patients and healthy individuals to human hsp60 and its peptides. J Autoimmun 12(2): 1999, 121-129.

24. Kipnis, J., Yoles, E., Porat, Z., Cohen, A., Mor, F., Sela, M., Cohen, I.R., Schwartz, M. T cell immunity to copolymer 1 confers neuroprotection on the damaged optic nerve: possible therapy for optic neuropathies. Proc Natl Acad Sci USA 97(13): 2000, 7446-7451.

25. Lacroix-Desmazes, S., Kaveri, S.V., Mouthon, L., Ayouba, A., Malanchere, E., Coutinho, A., Kazatchkine, M.D. Self-reactive antibodies (natura autoantibodies) in healthy individuals. J Immunol Methods 216(1-2): 1998, 117-137.

26. Paul, W.E. Affinity maturation. Fundam Immunol Chapter 25. (4th ed., 1998). 